\title{
Decreased Case Fatality Rate of COVID-19 in the Second Wave: a study in 53 countries.
}

\author{
Guihong Fan ${ }^{1}$, Zhichun Yang 2 , Qianying Lin $^{3}$, Shi Zhao ${ }^{4}$ Lin Yang ${ }^{5}$, and Daihai $\mathrm{He}^{4}$ \\ ${ }^{1}$ Columbus State University \\ ${ }^{2}$ Chongqing Normal University \\ ${ }^{3}$ University of Michigan \\ ${ }^{4}$ Affiliation not available \\ ${ }^{5}$ Hong Kong Polytechnic University
}

August 11, 2020

\begin{abstract}
The raw case fatality rate (CFR, reported number of COVID-19 deaths divided by the number of cases) is a useful indicator to quantify the severity or treatment efficacy in a locality. In many countries, the pandemic showed a two-wave pattern now, namely the daily reported cases once reached a low level and now went up. To our knowledge, no study has compared the CFR for the two waves. In this work, we report that in 53 countries or regions with the highest deaths, the CFR is reduced in 43 countries or regions in the on-going second wave. We discussed the possible reasons. Also, we compare the two-wave pattern of COVID-19 with the weekly influenza positive tests. The influenza activity in pre-pandemic era provided an indicator for climate in a country, since it is well-known that influenza is driven by weather. The sharp drop in 2020 influenza activity is an indicator of the effects of social distancing.
\end{abstract}

\section{Decreased Case Fatality Rate of COVID-19 in the Second Wave: a study in 53 countries}

Guihong Fan ${ }^{1}$, Zhichun Yang ${ }^{2}$, Qianying $\mathrm{Lin}^{3}$, Shi Zhao ${ }^{4,5}$, Lin Yang ${ }^{6}$, and Daihai $\mathrm{He}^{7, *}$

1 Mathematics Department, Columbus State University, US

2 School of Mathematical College, Chongqing Normal University, Chongqing, China, 401331

3 Michigan Institute for Data Science, University of Michigan, Ann Arbor, MI, United States

4 JC School of Public Health and Primary Care, Chinese University of Hong Kong, Hong Kong SAR, China 5 Shenzhen Research Institute of Chinese University of Hong Kong, Shenzhen, China

6 School of Nursing, Hong Kong Polytechnic University, Hong Kong, SAR, China.

7 Department of Applied Mathematics, Hong Kong Polytechnic University, Hong Kong Special Administrative Region, China

* Correspondence author: Daihai He, Department of Applied Mathematics, Hong Kong Polytechnic University, Hong Kong, China. E-mail: daihai.he@polyu.edu.hk;

Keywords: COVID-19; case fatality rate; influenza.

Running title: Time-varying case fatality rate of COVID-19

fan_guihong@columbusstate.edu 
yangzhch@126.com

qianying@umich.edu

zhaoshi.cmsa@gmail.com

l.yang@polyu.edu.hk

daihai.he@polyu.edu.hk

Abstract: The raw case fatality rate (CFR, reported number of COVID-19 deaths divided by the number of cases) is a useful indicator to quantify the severity or treatment efficacy in a locality. In many countries, the pandemic showed a two-wave pattern now, namely the daily reported cases once reached a low level and now went up. To our knowledge, no study has compared the CFR for the two waves. In this work, we report that in 53 countries or regions with the highest deaths, the CFR is reduced in 43 countries or regions in the on-going second wave. We discussed the possible reasons. Also, we compare the two-wave pattern of COVID-19 with the weekly influenza positive tests. The influenza activity in pre-pandemic era provided an indicator for climate in a country, since it is well-known that influenza is driven by weather. The sharp drop in 2020 influenza activity is an indicator of the effects of social distancing.

\section{Main Text}

The ongoing COVID-19 pandemic has caused a serious health threat globally. Many countries have seen a wave pattern of reported cases, namely a second wave followed the first wave. To our knowledge, there is no study to compare the case fatality rate between these two waves.
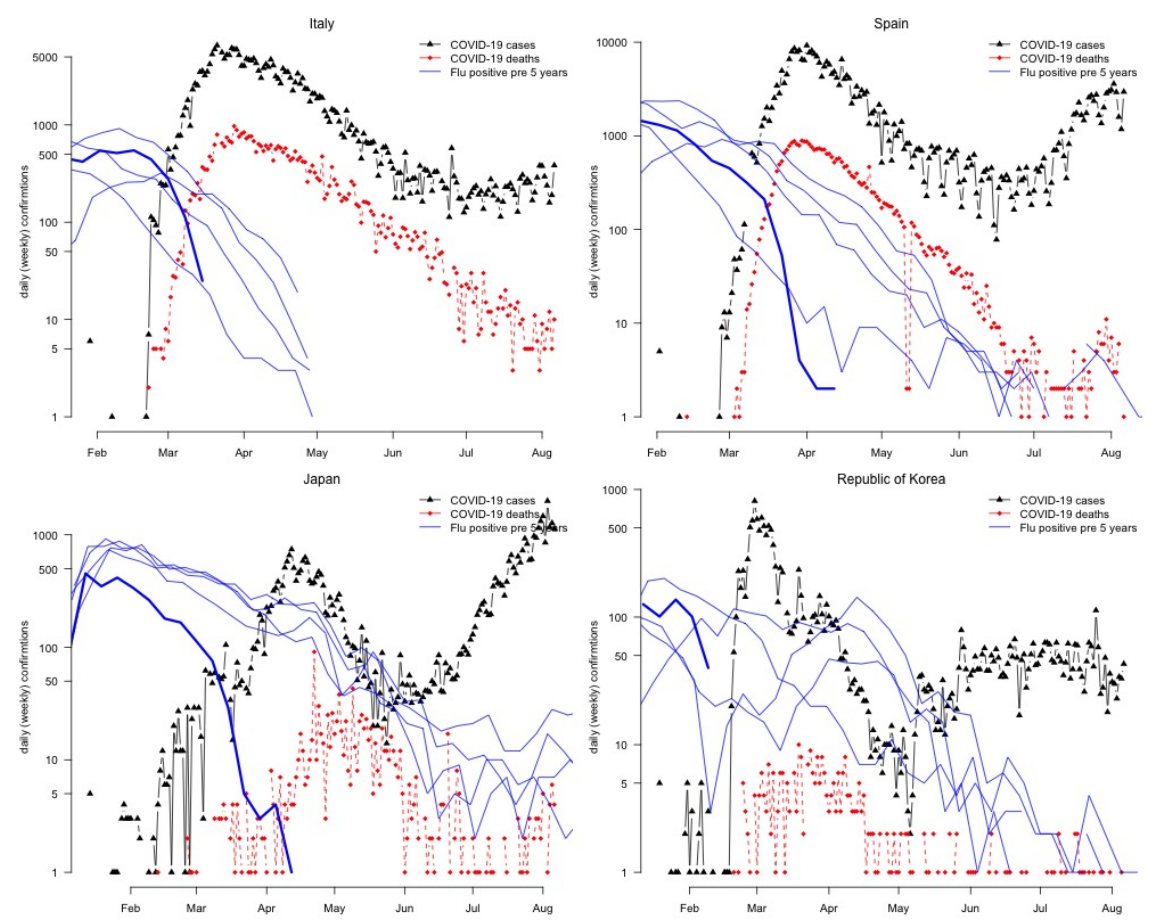

Figure 1. Time series plots of daily confirmed COVID-19 cases (in black), COVID-19 deaths (in red), and influenza positive cases in 2015-2019 (in light blue) and in 2020 (in dark blue).

We obtained the daily confirmations of COVID-19 cases and deaths for 216 countries or regions, during February 2020 - August 2020, and weekly influenza confirmations during 2015 to current week, from World Health Organization [1,2]. We showed data of four countries (two from Europe and two from Asia which 
caught a lot of attention in the early phase of the pandemic) in Figure 1. Research of other 49 countries or regions are listed in the supplementary. The time series (in common log scale on vertical axis) of confirmed cases showed two waves. Based on this observation, we divided the transmission of the disease into two phases: Phase I before June 1 and Phase II after June 1 for confirmed cases; and Phase I before June 10 and Phase II after June 10 for deaths. For deaths, we choose the truncated time ten days later to account for the delay between confirmation and deaths [3]. We compared the raw case fatality rate (CFR) of Phase I and Phase II for all countries or regions.

In our supplementary data in the appendix, for each country, we break down the data for Phase I or Phase II. The column "case_pre" is the total of confirmed cases before June 1 and the column "case_post" is the total of confirmed cases after June 1 up to July 26. Accordingly, the column of "death_pre" is the infection death before June 10 and the column "death_post" is the infection death after June 10 up to August 6. We define the raw case fatality rates $(\mathrm{CFR})$ as $r_{1}=\frac{\text { death_pre }}{\text { case_pre }}$ and $r_{2}=\frac{\text { death_post }}{\text { case_post }}$. Then the change in CFR is reduction $=\frac{r_{1}-r_{2}}{r_{1}}$. Based on our analysis, among all 53 hardest-hit countries or regions (supplementary Table 1), 43 of them had an apparent reduction in CFR. Only ten remaining countries or regions had an increase in CFR (negative reduction). The decrease in the CFR might indicate the severity of the global pandemic is becoming better. The potential reason for such decrease is worth further investigation. We propose the following hypotheses that could contribute to the decrease of CFR in the second phase. First, the apparent higher CFR in the first phase could be a harvest effect, namely a large number of elderly and individual with health conditions (the group at risk) likely died in the first phase, especially in these countries with a high infection rate, and this risk group run low in the second phase. If a country or region (such as Hong Kong) was spared from the first phase, of course it is not surprising to see an increase in CFR. Second, the age structure of infected changed due to a variety of reasons, e.g. social movement in many countries might involve more healthier young individuals. Third, the virus might evolve such that young health adults become more susceptible, thus lead to a reduced CFR. Forth, favourable climate might lead to reduced CFR (e.g. warmer weather in north hemisphere and improved air quality due to city lockdown [4-6]). Last but not least, improved timely treatment and enhanced massive testing could reduce the deaths and increase the number of cases, thus a reduced CFR in the second phase.

We show time series plots of eight countries in supplementary figure, where only Iran faces an increase in the raw CFR and a table summarizes results of 53 countries or regions. The weekly influenza laboratory confirmations for the previous five years may be used as a proxy of the weather, since it is well known that influenza seasonality is driven by temperature and humidity. Thus we may wonder whether favourable weather may contribute to a reduce CFR for COVID-19. The sharp drop in influenza cases in 2020 (dark bold curve), compared to previous years, may be due to social distancing and possible interference with COVID-10 infection. Thus it is informative to compare the COVID-19 and influenza in these plots. Individual data or age grouped data are needed to further clarify the phenomenon. The finding is nevertheless of significance to inform public and for policy making.

\section{Declarations}

\section{List of abbreviations}

COVID-19: coronavirus disease 2019

CFR: case-fatality-rate

\section{Ethics approval and consent to participate}

The ethical approval or individual consent was not applicable.

\section{Consent for publication}

Not applicable.

\section{Availability of data and materials}


All data and materials used in this work were publicly available.

\section{Conflict of interests}

DH was supported by an Alibaba (China) Co. Ltd. Collaborative Research project. Other authors declare no competing interests.

\section{Funding}

ZY was supported by National Natural Science Foundation of China under Grant 11971081, Science and Technology Research Program of Chongqing Municipal Education Commission, KJZD-M2020005. DH was supported by General Research Fund (15205119) of Research Grants Council of Hong Kong and Alibaba (China) Co. Ltd. Collaborative Research project. The funding agencies had no role in the design and conduct of the study; collection, management, analysis, and interpretation of the data; preparation, review, or approval of the manuscript; or decision to submit the manuscript for publication.

\section{Authors' Contributions}

All authors conceived the study, carried out the analysis, and drafted the first manuscript. All authors discussed the results, critically read and revised the manuscript, and gave final approval for publication.

\section{Acknowledgements}

Not applicable.

\section{References:}

[1] World Health Organization. https://covid19.who.int

[2] World Health Organization. https://www.who.int/influenza/gisrs_laboratory/flunet/en/

[3] Yang S, Cao P, Du P, Wu Z, Zhuang Z, Yang L, Yu X, Zhou Q, Feng X, Wang X, Li W, Liu E, Chen J, Chen Y, He D. Early estimation of the case fatality rate of COVID-19 in mainland China: A data-driven analysis. Annals of Translational Medicine. 2020. 8(4):128.

[4] Ran J, Zhao S, Han L, Chen D, Yang Z, Yang L, Wang MH, He D. The Ambient Ozone and COVID19 Transmissibility in China: A Data-Driven Ecological Study of 154 Cities. Journal of Infection. 2020. 81(3):e9-e11.

[5] Ran J, Zhao S, Han L, Qiu Y, Cao P, Yang Z, Chong MKC, Yang L, Wang MH, and He D. Effects of Particulate Matter Exposure on the Transmissibility and Case Fatality Rate of COVID-19: A Nationwide Ecological Study in China. Journal of Travel Medicine. 2020.

[6] Ran J, Zhao S, Han L, Liao G, Wang K, Wang MH, He D. A Re-analysis in Exploring the Association between Temperature and COVID-19 Transmissibility: An Ecological Study with 154 Chinese Cities. European Respiratory Journal. 2020. 01253.

\section{Supplementary figure}



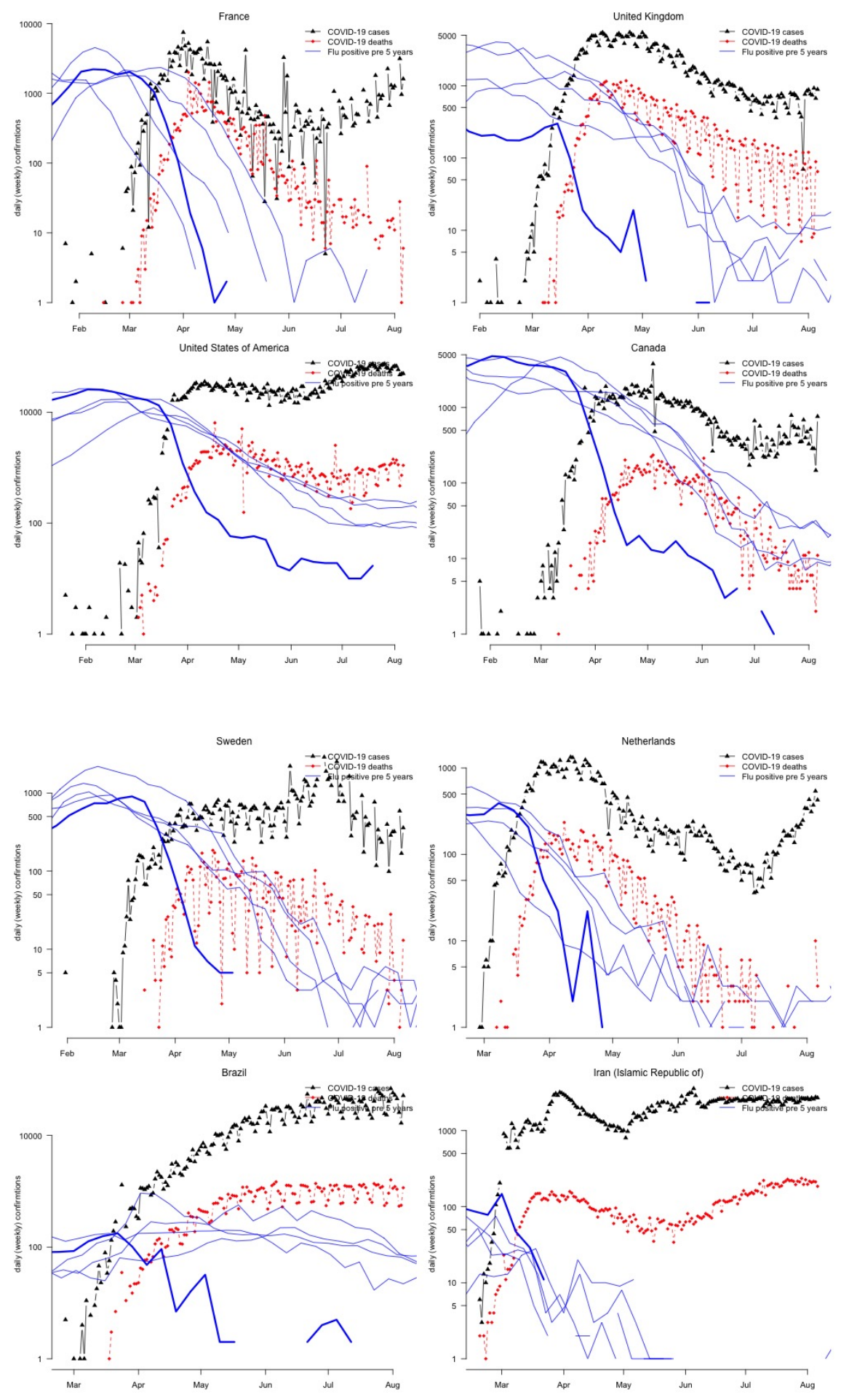

Supplementary Data by Aug. 6, 2020In the following table, for each country, we break down the data for Phase I or Phase II. The column "case_pre" is the data of confirmed cases before June 1 and the column "case_post" is the data of confirmed cases after June 1. Accordingly, the column of "death_pre" is the infection death before June 10 and the column "death_post" is the infection death after June 10. For the 
data after June, the end time of our data is Aug. 6, 2020. We define two raw case fatality rates as $r_{1}=\frac{\text { death_pre }}{\text { case_pre }}$ and $r_{2}=\frac{\text { death_post }}{\text { case_post }}$. In addition, we calculate the reduction in case fatality rate as reduction $=\frac{r_{1}-r_{2}}{r_{1}}$ and place the result in the last column. Table 1. The data breakdown for Phase I and Phase II

\begin{tabular}{|c|c|c|c|c|c|}
\hline $\begin{array}{l}\text { Country or } \\
\text { region }\end{array}$ & case_pre & death_pre & case_post & death_post & reduction \\
\hline $\begin{array}{l}\text { The United } \\
\text { Kingdom }\end{array}$ & 254394 & 40686 & 44042 & 5678 & 0.194 \\
\hline $\begin{array}{l}\text { United States } \\
\text { of America }\end{array}$ & 1734040 & 110220 & 2204054 & 45830 & 0.673 \\
\hline France & 148436 & 29149 & 19855 & 1033 & 0.735 \\
\hline Germany & 181482 & 8711 & 22701 & 464 & 0.574 \\
\hline Netherlands & 46257 & 6016 & 6147 & 137 & 0.829 \\
\hline Spain & 239650 & 28232 & 34846 & 267 & 0.935 \\
\hline Italy & 232664 & 33964 & 12674 & 1217 & 0.342 \\
\hline $\begin{array}{l}\text { Iran (Islamic } \\
\text { Republic of) }\end{array}$ & 148950 & 8351 & 135084 & 9451 & -0.248 \\
\hline Brazil & 465166 & 36455 & 1762348 & 59364 & 0.57 \\
\hline Sweden & 37113 & 4694 & 41650 & 1066 & 0.798 \\
\hline $\begin{array}{l}\text { Republic of } \\
\text { Korea }\end{array}$ & 11468 & 274 & 2511 & 28 & 0.533 \\
\hline Japan & 16851 & 916 & 11105 & 110 & 0.818 \\
\hline Switzerland & 30789 & 1676 & 3124 & 29 & 0.829 \\
\hline Belgium & 58751 & 9628 & 7117 & 231 & 0.802 \\
\hline Denmark & 11633 & 593 & 1757 & 23 & 0.743 \\
\hline Czechia & 9230 & 328 & 5570 & 60 & 0.697 \\
\hline Portugal & 32203 & 1485 & 17176 & 255 & 0.678 \\
\hline Austria & 16638 & 672 & 3484 & 47 & 0.666 \\
\hline Philippines & 18086 & 1011 & 56304 & 1112 & 0.647 \\
\hline China & 84570 & 4645 & 1930 & 39 & 0.632 \\
\hline $\begin{array}{l}\text { United Arab } \\
\text { Emirates }\end{array}$ & 33896 & 281 & 24092 & 74 & 0.629 \\
\hline Israel & 17071 & 295 & 39373 & 269 & 0.605 \\
\hline Algeria & 9267 & 715 & 16217 & 546 & 0.564 \\
\hline $\begin{array}{l}\text { Dominican } \\
\text { Republic }\end{array}$ & 16908 & 539 & 40707 & 683 & 0.474 \\
\hline Nigeria & 9855 & 361 & 29093 & 566 & 0.469 \\
\hline Argentina & 14702 & 670 & 127198 & 3339 & 0.424 \\
\hline Ecuador & 38571 & 3642 & 39577 & 2205 & 0.41 \\
\hline Canada & 89741 & 7800 & 22499 & 1158 & 0.408 \\
\hline Pakistan & 69496 & 2172 & 200904 & 3863 & 0.385 \\
\hline Turkey & 163103 & 4711 & 60212 & 1073 & 0.383 \\
\hline South Africa & 30967 & 1080 & 377085 & 8218 & 0.375 \\
\hline Poland & 23571 & 1166 & 18009 & 590 & 0.338 \\
\hline Hungary & 3867 & 548 & 531 & 51 & 0.322 \\
\hline Bangladesh & 47153 & 930 & 168957 & 2337 & 0.299 \\
\hline India & 182143 & 7466 & 1105802 & 33233 & 0.267 \\
\hline Indonesia & 26473 & 1883 & 67184 & 3569 & 0.253 \\
\hline $\begin{array}{l}\text { Republic of } \\
\text { Moldova }\end{array}$ & 8098 & 359 & 14007 & 464 & 0.253 \\
\hline Ukraine & 23672 & 810 & 39151 & 1009 & 0.247 \\
\hline
\end{tabular}




\begin{tabular}{llllll}
\hline $\begin{array}{l}\text { Country or } \\
\text { region }\end{array}$ & case_pre & death_pre & case_post & death_post & reduction \\
\hline Romania & 19133 & 1334 & 22142 & 1187 & 0.231 \\
Sudan & 4800 & 372 & 6502 & 391 & 0.224 \\
Mexico & 84627 & 13699 & 277647 & 35170 & 0.217 \\
Iraq & 6179 & 370 & 96047 & 4724 & 0.179 \\
Panama & 12531 & 393 & 43375 & 1129 & 0.17 \\
Bolivia & 8731 & 465 & 55404 & 2855 & 0.032 \\
(Plurinational & & & & & \\
State of) & & & & & \\
Egypt & 23449 & 1271 & 66964 & 3660 & -0.008 \\
Colombia & 26688 & 1259 & 191740 & 10056 & -0.112 \\
Chile & 94858 & 2264 & 243901 & 7528 & -0.293 \\
Ireland & 24929 & 1683 & 897 & 80 & -0.321 \\
Russian & 405843 & 6142 & 395006 & 8464 & -0.416 \\
Federation & & & & & \\
Saudi Arabia & 83384 & 746 & 177010 & 2274 & -0.436 \\
Afghanistan & 15094 & 376 & 20887 & 922 & -0.772 \\
Peru & 148285 & 5465 & 218265 & 14542 & -0.808 \\
Belarus & 41658 & 276 & 25030 & 301 & -0.815 \\
\hline
\end{tabular}

